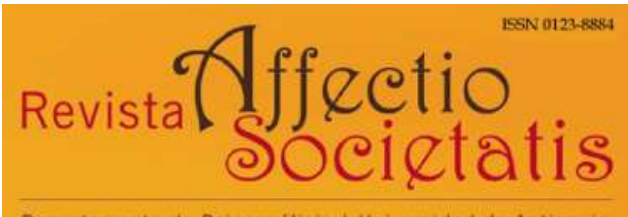

Departamento de Psicoanálisis | Universidad de Antioquia

Revista Affectio Societatis

Departamento de Psicoanálisis

Universidad de Antioquia

revistaaffectiosocietatis@udea.edu.co

ISSN (versión electrónica): 0123-8884

Colombia

\author{
2019 \\ María Catalina León Suárez \\ CUANDO SE APRENDE NADA: UN DIÁLOGO ENTRE LOS \\ PROBLEMAS DE APRENDIZAJE Y LA ANOREXIA MENTAL EN \\ PSICOANÁLISIS \\ Revista Affectio Societatis, Vol. 16, N. ${ }^{\circ}$ 30, enero-junio de 2019 \\ Art. \# 4 (pp. 83-96) \\ Departamento de Psicoanálisis, Universidad de Antioquia \\ Medellín, Colombia
}




\title{
CUANDO SE APRENDE NADA: UN DIÁLOGO ENTRE LOS PROBLEMAS DE APRENDIZAJE Y LA ANOREXIA MENTAL EN PSICOANÁLISIS
}

\author{
María Catalina León Suárez ${ }^{1}$ \\ Universidad de Buenos Aires, Argentina \\ mariacatalinaleonsuarez@gmail.com \\ ORCID: 0000-0002-7289-482X
}

DOI: 10.17533/udea.affs.v16n30a04

\section{Resumen}

Este artículo presenta los resultados de una investigación sobre la clínillo del concepto nada y se abordan otras nociones importantes para el ca de los problemas de aprendizaje desde un abordaje psicoanalítico en un diálogo con la clínica de la anorexia mental. Se destaca que, similar a lo que ocurre con la anorexia, en la que se come nada, en algunos problemas de aprendizaje no se trata de que no se aprenda, sino de que se aprende nada; razón por la que se hace un desarropsicoanálisis, entre las que están el deseo, la falta, el amor y el objeto a. Esto se ejemplifica con algunos casos, siendo el principal de ellos el de Ernest Kris, comentado por Lacan, "El hombre de los sesos frescos".

Palabras clave: problemas de aprendizaje, anorexia mental, nada, falta, deseo.

1 Licenciada en psicología y pedagogía, psicoanalista infantil y de adolescentes, interesada en el trabajo con población vulnerable, en ese sentido en el apoyo a propuestas de fortalecimiento personal y comunitario para la generación de procesos de paz y sana convivencia. Específicamente vinculada a la educación en el estudio y abordaje de las dificultades de aprendizaje, orientada a la investigación sobre éstas, al apoyo en la construcción del currículo y la formulación de la evaluación educativa. 


\section{LEARNING NOTHING: A DIALOG BETWEEN LEARNING DISABILITY AND MENTAL ANOREXIA IN PSYCHOANALYSIS}

\begin{abstract}
This paper presents the results of a research on the clinic of learning disability from a psychoanalytic approach in a dialog with the clinic of mental anorexia. It highlights that, similar to anorexia, in which nothing is eaten, in some learning disabilities the problem is not the impossibility to learn, but the fact that nothing is learned. For this reason, we develop nothingness as a
\end{abstract}

concept and address other notions important to psychoanalysis, such as desire, lack, love, and object a. Some cases are used as an illustration of this; the main of them is the one of Ernest Kris, commented by Lacan, "the man of the fresh brains".

Keywords: learning disability, mental anorexia, nothing, lack, desire.

\section{LORSQUE L'ON N'APPREND RIEN : UN DIALOGUE ENTRE LES TROUBLES D'APPRENTISSAGE ET L'ANOREXIE MENTALE EN PSYCHANALYSE}

\section{Résumé}

Cet article présente les résultats d'une recherche sur la clinique des troubles d'apprentissage sous un angle psychanalytique, en établissant un dialogue avec la clinique de l'anorexie mentale. Il en ressort que, de manière similaire à ce qui survient avec l'anorexie lorsque l'on ne mange rien, dans quelques troubles d'apprentissage il ne s'agit pas de ne pas apprendre, mais de ne rien apprendre. Cela mène à dé- velopper le concept de rien et à aborder d'autres notions importantes pour la psychanalyse, notamment celles de désir, manque, amour et objet a. L'on illustre cela par quelques exemples, en particulier celui de Ernest Kris, commenté par Lacan, « L'homme aux cervelles fraîches ».

Mots-clés : troubles d'apprentissage, anorexie mentale, rien, manque, désir.

Recibido:04/10/2017• Aprobado:7/05/2018 


\section{Introducción}

El presente artículo desarrolla de manera breve el recorrido de un trabajo investigativo llevado a cabo durante la realización de la Maestría en Psicoanálisis de la Universidad de Buenos Aires, que parte de una inquietud sobre la creciente sintomatología de los problemas para aprender y sus asociados desánimo escolar, dificultad para estar en el espacio escolar y con los otros; así como de una escucha activa de tal fenómeno, que lleva a pensar si hay un más allá en el hecho de no comprender, de no aprender, que pueda tener algún diálogo con lo que ocurre en las anorexias mentales y el aparente hecho de no comer.

Tales inquietudes hacen síntesis en la pregunta orientadora de la investigación, ¿de qué manera es posible considerar, desde el psicoanálisis, una propuesta de abordaje de algunos problemas de aprendizaje a partir de un diálogo entre la clínica de estos y la de las anorexias mentales?, puesto que se parte de la hipótesis de que algunos de los llamados "problemas de aprendizaje" son un paradigma de los efectos de la nada como objeto en las relaciones establecidas por el sujeto -particularmente con el aprendizaje-, cuando la función de la falta no ha conseguido forjarse a cabalidad; lo cual se puede constatar estableciendo un diálogo entre la lectura de Jacques Lacan sobre la anorexia, y los problemas de aprendizaje.

Los objetivos de la investigación fueron, entonces, establecer la relación existente entre la estructuración subjetiva y el saber -como base del aprendizaje-, y el diálogo que frente a ello es posible entablar con algunas anorexias; realizar un diálogo entre los planteamientos fundamentales de la clínica de las anorexias y el saber articulado con el aprendizaje, para argumentar una propuesta de abordaje de algunos problemas de aprendizaje; y estudiar el caso de Ernest Kris, "El hombre de los sesos frescos", y la lectura que Jacques Lacan hace de éste, como ilustración de la tesis, ya que en su análisis se ponen en diálogo la inhibición intelectual y la posición anoréxica.

Como puede colegirse de lo anterior, el método investigativo utilizado es el diálogo, como garante de que las diferentes fuentes, incluidos los documentos teóricos, las experiencias clínicas y también 
los distintos conceptos desde la apertura teórica que cada uno implica, encuentren un espacio común, abierto a los aportes, a los puntos de lanza que cada uno sitúa.

\section{Constitución subjetiva y saber}

Los problemas de aprendizaje interrogan por el saber: ¿el estudiante sabe o no', ¿puede llegar a saber?; pero cuestionarse sobre lo que esto realmente implica lo inspira Freud, quien tempranamente sustenta en los albores del sujeto la manera como se forja la curiosidad: en la investigación sexual infantil ve el comienzo del apetito por saber y la pulsión de investigar, todo ello en una íntima relación con la estructuración subjetiva. Lo anterior lleva a preguntarse, ¿acaso el saber que se pone en juego dentro de la escuela, que muchas veces no logra ser aprendido, tiene alguna relación con el saber que está en la base de la estructuración subjetiva? y ¿de qué se trata ese saber primigenio?; así como también por la relación entre anorexia, alimentación y saber, como puntos de anclaje a partir de los cuales se establezcan algunas lecturas frente a los problemas de aprendizaje.

En "Un recuerdo infantil de Leonardo da Vinci" (2003/1910), Freud establece una importante relación entre la sexualidad infantil y el saber: el insaciable placer por preguntar que caracteriza a los niños es el punto del que parte, y lo reconoce como testimonio del apetito de saber de aquellos que comienzan a vivir: sexualidad y apetito en la base del saber. Appetere significa anhelar, desear ardientemente (Collins English Dictionary, 2015); Freud lo usa para referirse al saber, mas es por excelencia aquello que remite al alimento, a una necesidad corporal que implica la comida o la bebida, y es también lo que se pone en entredicho en la anorexia: al parecer al sujeto con anorexia le apetece nada, ¿desea ardientemente nada?; y en el caso del saber, cuando no se logra acceder al saber escolar, ¿se apetece nada sobre el saber, se está saciado? Piera Castoriadis Aulagnier da unas pistas iniciales para comprender el apetito más allá de la necesidad corporal, como oferta de sentido y fundamento para el deseo, pues “(...) en las primeras moléculas de leche que toma el infans: el aporte alimenticio 
se acompaña siempre con la absorción de un alimento psíquico que la madre interpretará como absorción de una oferta de sentido" (Aulagnier, 2010, pp.38-39).

Así, el saber que se juega en aquel instante primigenio es el del acceso al mundo de los humanos por medio del baño de lenguaje que describe Lacan, pero también es el que nombra Aulagnier como un don necesario para la vida del infans. Este don es el que está en la base de la frustración e implica que la pérdida introduce la falta, esto es, que sobre el fondo del signo de amor ha habido primero una anulación para reaparecer luego como pura presencia. Entonces, un saber fundante para el sujeto hace su aparición, a partir de él la satisfacción de la necesidad sufre una transformación y adquiere un valor simbólico la actividad en que se pone en juego el objeto de la necesidad; en lo que tiene que ver con la alimentación implica que esta, al ser don y símbolo, no es una incesante demanda materna para suplir lo que sería una pura necesidad -como ocurre con la anorexia-, y también para los problemas de aprendizaje, no es una estricta demanda para aprender los contenidos académicos. Desconocer el valor simbólico de alimentarse y de aprender es taponar la falta que vehicula el deseo para que éstos sean libidinizados por el sujeto.

Es la razón por la que Lacan enseña que en la anorexia no se trata de que el sujeto no coma, sino de que come nada; no es que no desee, hay un deseo débil, es un deseo de nada; la nada antepuesta en la relación con el otro que le ha atiborrado es el medio por el cual busca dar paso a una falta que le ha sido taponada. Pero en algunos problemas de aprendizaje, tal atiborramiento ocurre de manera similar, y los diferentes profesionales al servicio de la educación y la escuela, iniciando con el maestro, así como los padres e incluso el sistema mismo, taponan la falta para desear saber, exigiendo el estricto cumplimiento del plan de estudios tal y como ha sido diseñado; además ostentan un saber especializado sobre lo que supuestamente le ocurre al sujeto, las razones por las que no aprende y lo que debe hacerse al respecto.

Puede decirse, entonces, que hay igualmente una dificultad para acceder al mundo simbólico del saber en la relación con los otros, 
pues ya todo se sabe y está dicho, lo cual tapona el deseo, el amor por el saber, y sitúa algunos casos en los que, como ocurre con la anorexia, el sujeto antepone la nada en la relación con el Otro, y entonces no se trata de que no aprenda, se trata de que aprende nada.

Los momentos primeros de la existencia y la estructuración subjetiva de que se ha venido hablando, suponen también el paso fundamental por el Edipo y la castración que, a su vez, implican un saber sobre el cual se define la manera en que el sujeto se asumirá en el mundo; como lo plantea Mario Elkin Ramírez (2007), el saber introyectado de la ley significante, la normatividad y la afirmación que le permiten al sujeto establecer juicios de existencia sobre las cosas y de atribución sobre la realidad, que en el campo de la educación escolar y del aprendizaje se traduce en la posibilidad de estar con los otros, de asumirse en una institución que por excelencia recrea las normas que permiten estar en lo social y la manera en que le es posible posicionarse respecto del aprendizaje con el alcance simbólico que tiene éste.

Se trata, de esta manera, de una relación con el saber que, por supuesto, es simbólica y que tiene efectos sobre el cuerpo, aquel que entonces no es el meramente orgánico, sino que está afectado por el lenguaje, en un efecto que Lacan ha nombrado -a partir del estudio que hace de los estoicos- como incorporación: lo simbólico es un incorporal que toma cuerpo, a través de lo cual es posible ubicar la presencia de las cosas en el mismo.

Entonces, el cuerpo como organismo no es garantía de una inscripción simbólica, es necesaria una incorporación que implica el alimento y el aprendizaje, y que consiste en la transmisión de ese saber sobre la falta y la castración, sobre el Padre Muerto (un saber que proviene de la función materna). También es necesaria la identificación primaria que implica la pérdida del objeto $a$, y con ello el nacimiento de la pulsión, del agujero alrededor de la nada que instala un modo pulsional del comer, incluso del aprender, gracias al cual, por ejemplo, las comidas se hacen más apetecibles y hay una curiosidad y un gusto por saber más. Éste saber que se transmite está también presente en la relación con otros representantes del Otro, como los 
maestros, quienes de la misma manera transmiten su falta y con ello, su amor por el saber.

\section{Las anorexias y el saber, a propósito de algunos problemas de aprendizaje}

Sigmund Freud no hace un amplio desarrollo investigativo sobre la anorexia, especialmente porque la vincula, en el trabajo con casos clínicos, con la histeria, donde se hace presente como síntoma importante. Una de las menciones que realiza se encuentra en "Un caso de curación por hipnosis", donde plantea varios desarrollos clínicos a los que se refiere como "observaciones sobre la génesis de síntomas histéricos por 'voluntad contraria'" (Freud, 2001/1892-1893, p.147), una voluntad contraria que puede ser una expresión muy primigenia del "come nada" que señala Lacan, en lugar del "no come" para los casos de anorexia; lo que convoca a pensar en el campo de la educación un "aprende nada", en lugar del "no aprende" para algunos casos de problemas de aprendizaje, pues la experiencia en el abordaje de estos ha mostrado que no se trata de una voluntad que no se tiene, se trata de una voluntad contraria que se evidencia en el carácter de división subjetiva que está en la acción y no en la negación consciente, y que es contraria en la medida en que se juega inexorablemente en la relación con un Otro que ha ubicado su voluntad por encima del ser del sujeto estando ahí, siempre presente y con certezas sobre éste.

Freud relata algunos casos de "voluntad contraria", y específicamente el de una de sus pacientes, Emmy Von N. (1999); ante la negativa de involucrarse subjetivamente en el propio padecimiento, Freud le exige reevaluar su situación so pena que continúen en el tratamiento, lo cual tiene un efecto curador en la mujer. La postura que al respecto toma Freud es interesante y reveladora, es una postura que podría considerarse amorosa, en la que hay unas condiciones para la presencia de Freud en el tratamiento: él puede faltar y no está dispuesto a ser ubicado en el mismo lugar en que esta mujer ubica a las personas que le rodean. Solo a través de la falta es que florece el deseo y es esta la posición amorosa que enseña Freud; pero muchos 
casos de anorexia y de problemas de aprendizaje lo que revelan es un Otro siempre presente e implacable.

Varias de las referencias de Lacan a la anorexia sitúan este punto fundamental:

(...) frente a lo que tiene delante, es decir, la madre de quien depende, hace uso de esa ausencia que saborea. Gracias a esta nada, consigue que ella dependa de él. Si no captan esto, no pueden entender nada, no sólo de la anorexia mental, sino también de otros síntomas. (Lacan, 1999/1956-1957, p.187).

(...) pero el niño no se duerme siempre así en el seno del ser, sobre todo si el Otro, que a su vez tiene sus ideas sobre sus necesidades, se entromete, y en lugar de lo que no tiene, le atiborra con la papilla asfixiante de lo que tiene, es decir confunde sus cuidados con el don de su amor. (Lacan, 2002, p.608).

Entonces, una posición de absoluta presencia resulta asfixiante y $a-t i-b o r r a$ porque confunde la necesidad con el don de amor. La pura necesidad en la alimentación es la que lleva a concebir el cuerpo como un mero organismo y no permite que el alimento sea libidinizado; la necesidad respecto al saber y en el aprendizaje es lo que lleva a que se memoricen solo contenidos, se cumpla estrictamente un plan de estudios que debe aprenderse de la misma forma para todos, se enseñe y evalúe bajo las premisas deterministas del método científico.

Contrario a ello, el don de amor vehiculiza el deseo porque implica dar lo que no se tiene; que todo no está dicho, que no hay completa satisfacción, implica la falta. La respuesta a la omnipotencia en la confusión entre necesidad y deseo es, como lo señala Lacan (1999/1956-1957), bajo el signo de la nada, pues es la manera como el sujeto consigue anteponer, separar en la relación con el Otro y, fundamentalmente, proteger el deseo propio. Es una disposición lógica y ética del sujeto hacia el Otro, forma en que suple el vacío, y objeto separador del Otro como apertura al enigma del deseo en éste, provocando la falta en la cual le es posible inscribirse. Sin embargo, el deseo en la anorexia no llega a ser lo suficientemente fuerte, y como señala Recalcati, esta disposición ética se queda coja, no vale como imperati- 
vo porque el deseo se revela débil, pues aun cuando el sujeto se vacía para salvaguardarlo, no encuentra cómo asumirlo y por ello es "un hundimiento sobre el propio deseo. Es un empobrecimiento progresivo del deseo. Es un derrumbe del deseo" (Recalcati, 2004, p.6).

La absoluta presencia que ha implicado un derrumbe del deseo es definida por Lacan al referirse al deseo de la madre y a la anorexia como un estrago, es una devoración, un vínculo desesperado, un devastamiento. En lugar de que el hijo ocupe una posición que divida a la madre, que la haga desear más allá de éste, ha sido llamado a colmarla, quedándose supeditado al retorno de la demanda de amor, esto es, a la relación en la cual no hay un límite, en la que en lugar de darse lo que no se tiene como muestra de amor, se da todo a expensas del goce absoluto. La anorexia, por excelencia, presenta esta relación dramática cuyo estrago muy bien describe Recalcati:

(...) para obtener el signo de amor, el sujeto puede llegar al extremo de consumir todo su ser, puede hacer de su ser un cúmulo de ruinas (...) la búsqueda y la demanda infinita del signo de amor pueden invertirse en su contrario, resistente a toda dialéctica. Puede devenir estrago, odio puro, rechazo sin apelación del Otro. (Recalcati, 2004, pp.134-135).

Ahora bien, ¿hasta qué punto tal devastación en la relación con el Otro -que llega incluso a suponer un cierto arrasamiento de la dimensión subjetiva- se evidencia también en la relación patológica de algunos sujetos con el ámbito escolar? Al ser la escuela por excelencia un mundo de vínculos simbólicos, los aprendices, desde la particularidad, pero especialmente aquellos que tienen fuertes dificultades para estar en la escuela y en el proceso académico, cuestionan fuertemente las distintas relaciones que se entablan con el Otro; ya sólo la excesiva inquietud, la imposibilidad de controlar el cuerpo, el maestro angustiado, la cotidianidad escolar agitada, el sujeto descontrolado y sin un lugar en la institución, puede llegar a catalogarse como un verdadero estrago.

Si se examina con detenimiento el Otro de la dificultad escolar, del llamado fracaso escolar, puede llegar a tener unas características 
similares a las del Otro en la anorexia; es un efecto estragante por una presencia persecutoria que el sujeto vive, y que en la escuela puede multiplicarse en la función que cumplen los maestros, los padres, los terapeutas, el sistema mismo..., todos representantes del Otro; un efecto estragante del que tampoco se escapan algunos maestros, quienes con la vestidura simbólica de su labor más que desquebrajada parecen padecer cada día su función.

\section{Saber, goce y cuerpo: marcas del significante. Efectos en las anorexias y en los problemas de aprendizaje}

El caso de Ernest Kris, "El hombre de los sesos frescos", leído y estudiado por Lacan, es un ejemplo clínico del diálogo entre el campo académico, de las dificultades para aprender y la anorexia mental. En los Escritos 2, Lacan señala, refiriéndose a la interpretación que hace Kris del caso:

Trata usted al paciente como a un obsesivo, pero él le tiende la pértiga con su fantasía de comestible: para darle la sensación de adelantarse en un cuarto de hora a la nosología de su época diagnosticando: anorexia mental. Refrescará usted de pasada, devolviéndolo a su sentido propio, ese par de términos reducidos por su empleo corriente a la dudosa calidad de una indicación etiológica(...) anorexia, en este caso, en cuanto a lo mental, en cuanto al deseo del que vive la idea, y esto nos lleva al escorbuto que reina en la balsa en la que lo embarco con las vírgenes flacas. (Lacan, 2002, p.581).

Esta afirmación, al igual que las demás hechas por Lacan cuando se refiere a la interpretación y a la anorexia, resulta polémica y acertada, dado que marca una manera tajantemente distinta de pensar y direccionar la problemática con la alimentación y la interpretación misma, poniendo en cuestión cómo son abordadas por la ego-psychologie, de la cual es representante Ernest Kris.

El caso se trata de un joven científico de unos treinta años que ocupa con éxito una posición académica respetable, sin poder alcan- 
zar una más elevada por ser incapaz de publicar sus amplias investigaciones, pues tiene, según él, un impulso a robar las ideas de los otros, en especial las de un amigo con el que conversa a menudo. Para Ernest Kris en la dirección de la cura fue necesario ir a las pautas de comportamiento del paciente, las conductas típicas, que en últimas revelan las actividades defensivas del yo, y reconocer en ellas una "alianza" para la cura, razón por la que se encarga de averiguar en profundidad sobre un texto que el paciente dice plagiar, con el fin de demostrarle que esto no es así.

La respuesta que recibe es la que comenta Lacan en la referencia arriba citada, el paciente al escuchar contradecir su hipótesis, después de un largo silencio afirma: "Todos los días al medio día, cuando salgo de aquí, antes del almuerzo, y antes de volver a mi oficina, me paseo por la calle $\mathrm{x}$ (una calle bien conocida por sus pequeños y atractivos restaurantes) y miro los menús detrás de las vidrieras. Es en uno de esos restaurantes donde encuentro de costumbre mi plato favorito: sesos frescos" (Kris, 1998/1924, p.42).

Una respuesta que, sin lugar a dudas es una reafirmación sobre la propia verdad negada por Kris, y a la cual, con el tono sarcástico que lo caracteriza, Lacan se refiere así: “(...) esa mostaza después de cenar que el paciente respira, me parece que dice más bien al anfitrión que faltó durante la cena. Por muy compulsivo que sea para olfatearla, se trata de un hirt; síntoma transitorio, sin duda, advierte al analista: erró usted el blanco". (Lacan, 2002, p.580). ¿El anfitrión faltó durante la cena?, ¿erró usted en el blanco?, Kris ¿embarcado en la balsa con las vírgenes flacas?, hay claramente para Lacan un cuestionamiento a la posición del analista, a la manera cómo interpreta el caso, revelando con ello un gran error: el síntoma es negado por el analista al aplicar la técnica "de la superficie a la profundidad", y como respuesta el paciente produce un acting out; la manera como Lacan argumenta esto es entablando un diálogo con la anorexia mental, remarcando que el énfasis está, tal como ocurre con los sujetos que tienen anorexia, en $e l$ deseo del que vive la idea.

Se está aquí frente a un caso de inhibición intelectual, de estancamiento ante la posibilidad de avanzar en el ámbito académico que tie- 
ne mucha importancia para el paciente, un caso que, en esa medida, es, como ya se había mencionado, similar a otros que ocurren y que son nombrados como problemas de aprendizaje. Podría decirse que este paciente se reconoce a sí mismo con problemas para aprender, en la medida en que dice con sus palabras y también con su acting no poder fabricar una idea propia y siempre estar usurpando las de los demás para reconocer su validez. En otros casos de problemas de aprendizaje se encuentra algo cercano, hay en estas problemáticas la tendencia a pensar que definitivamente no se puede aprender, y que es el otro quien logra hacer siempre todo correctamente, además de otras características asociadas, como el aparente desinterés académico y el registro de todo un historial de análisis frente a la dificultad de parte de distintos profesionales que, como Kris, tienen "la verdad" sobre lo que le pasa al sujeto.

Son varias las enseñanzas que permite ver el diálogo entablado por Lacan en este caso, y que se complementan igualmente en un caso presentado sobre un paciente que, similar al de Kris, no es que no aprenda, es que aprende nada. En el caso comentado por Lacan, se ve al paciente recurrir al robo, al plagio, para tomar distancia del Otro y sacar de su alcance los objetos a través de los cuales se relaciona; en el Otro caso, el paciente hace recurso a la mentira, justo cuando el valor de la honestidad es el más preciado por su madre y anteponerla así en la relación estragante que ha entablado con el Otro paterno y materno, e incluso el Otro docente.

\section{Algunas conclusiones}

Algunas de las conclusiones que la investigación permite establecer atendiendo al problema investigativo son que el mecanismo que opera en el alimentarse y en el aprender es semejante debido a que penden de un apetito que emerge de la relación con el Otro; de ahí que esta relación sea tan importante y requiera de la falta como estructural, aquella que es transmitida por el Otro materno, como posibilidad de ingreso al mundo simbólico y de límite al goce, siendo la razón por la que comer, saber y aprender tienen su origen en la sexualidad misma. 
Alimentarse y aprender tienen igualmente fundamento en la sexualidad porque están entrelazados con una satisfacción libidinosa de la oralidad proveniente del investimento pulsional del Otro; esto implica al aprendizaje, por cuanto a través de la alimentación la madre transmite el mundo simbólico, fundamento de los procesos del pensamiento, e involucra de la misma forma al vacío, porque ningún alimento y ninguna atención proporcionan una satisfacción completa, siendo la antítesis de ello los excesos en los cuidados de una madre siempre presente, como ocurre en las anorexias, en las que la madre está presta a colmar y borrar la falta.

Una de las consecuencias de este fundamento en la sexualidad es que la relación con el saber es en principio por un rechazo activo, ya que la investigación intelectual inicial es sobre la sexualidad y, por esta razón, pasa a ser rápidamente reprimida, luego a estar tomada por la falta como parte de los descubrimientos de esa investigación, la cual da apertura al deseo de saber.

Ahora bien, así como la relación con el alimento y el aprendizaje implican la sexualidad, requieren en su fundamento de un don, de un signo del amor del Otro, pues el amor es el que está habitado por la falta; y entonces, porque no es completo, se quiere más, porque no es completo siempre hay un don que entregar, porque es carente se apetece más, porque es enigmático se busca más; igualmente ocurre con el saber, de ahí que un plan de estudios acabado e inamovible, un maestro siempre presente que lo sabe todo, unos padres siempre acompañantes... sean la antítesis del amor al saber y el fondo de una nada como rechazo absoluto.

La nada, de esta manera, es el objeto introducido por el sujeto en la relación con el Otro, por ello es una disposición lógica y ética; es la forma en que se suple el vacío llenado y se hace apertura al enigma de un deseo que no alcanza a ser lo suficientemente fuerte para que la posición del sujeto, en la anorexia y en los problemas de aprendizaje, sea menos mortífera. Contrario a esto, es preciso una relación con el Otro en la que haya un punto de no-coincidencia que permita articular una diferencia, es a partir de ello que el Otro no es solamente el del tener: el que posee el alimento, el saber, sino el que le falta. 
Finalmente, puede decirse que el caso de "El hombre de los sesos frescos" brinda un ejemplo claro sobre la importancia de la posición del analista y su interpretación, enseñanza que se extiende al campo educativo en el papel que le corresponde al maestro y a los diferentes profesionales presentes en él, llevando a recordar, entre otros aspectos, que la posición que asumen estos actores es determinante y puede llegar a tener un efecto estragante en el aprendiz -similar al que ocurre en las anorexias-, y que hay un más allá en las problemáticas presentes: un anclaje de goce del que pende la repetición de la dificultad y que permite entender por qué algunos problemas de aprendizaje se sobreponen a meras estrategias reeducativas y de enseñanza.

\section{Referencias bibliográficas}

American Psychological Association. (2015). Collins English Dictionary. Dictionary.com. Recuperado de: http://dictionary.reference.com/browse/appetite

Aulagnier, P. (2010). La violencia de la interpretación. Del pictograma al enunciado. Buenos Aires, Argentina: Amorrortu.

Freud, S. (2003/1910). Un recuerdo infantil de Leonardo da Vinci. En J. Strachey (Ed.) y J. L. Etcheverry (trads.). Obras completas (Vol. XI). Buenos Aires, Argentina: Amorrortu.

Freud, S. (1999/1893-1895). Estudios sobre la histeria. En J. Strachey (Ed.) y J. L. Etcheverry (trads.). Obras completas (Vol. II). Buenos Aires, Argentina: Amorrortu.

Freud, S. (2001/1892-1893). Un caso de curación por hipnosis. En J. Strachey (Ed.) y J. L. Etcheverry (trads.). Obras completas (Vol. I). Buenos Aires, Argentina: Amorrortu.

Kris, E. (1998/1924). La psicología del yo y la interpretación en la terapia psicoanalítica. Textos de referencia. Publicación interna de la Asociación de Psicoanálisis. Barcelona, España: Biblioteca Freudiana de Barcelona.

Lacan, J. (2002). Escritos 2. Buenos Aires, Argentina: Siglo Veintiuno Editores.

Lacan, J. (1999/1956-1957). Seminario, Libro 4: La relación de objeto. Buenos Aires, Argentina: Paidós.

Ramírez, M. (2007). Psicoanálisis con niños y dificultades en el aprendizaje. Medellín, Colombia: Editorial Universidad de Antioquia.

Recalcati, M. (2004). La última cena: anorexia y bulimia. Buenos Aires, Argentina: Ediciones del cifrado. 